\title{
Ultrasonic Enhancement of Photoacoustic Emissions by
}

\section{Nanoparticle-Targeted Cavitation}

\author{
James R. McLaughlan, ${ }^{1, *}$ Ronald A. Roy, ${ }^{1}$ Hengyi Ju, ${ }^{2}$ and Todd W. Murray ${ }^{2}$ \\ ${ }^{1}$ Boston University, Department of Mechanical Engineering, 110 Cummington Street, \\ Boston, MA 02215 \\ ${ }^{2}$ University of Colorado at Boulder, Department of Mechanical Engineering, 427 UCB \\ Engineering Center, Boulder, CO 80309 \\ *Correspondingauthor: jmcl@bu.edu
}

A technique to enhance the formation of micro-bubbles around nanoparticles is presented. Gold nanoparticle-doped phantoms are subjected to pulsed optical and ultrasound fields, resulting in bubble formation and collapse, and producing strong acoustic emissions. The applied ultrasound field allows for cavitation at lower laser fluencies than using light alone. The acoustic emission associated with bubble collapse well exceeds the direct photoacoustic response, and is used to image a nanoparticledoped region in a tissue phantom. The threshold fluence reduction afforded by ultrasound assisted cavitation, and the associated acoustic emission, make the technique well suited for nanoparticle-targeted biological imaging and tissue therapy.

OCIS codes: $170.5120,110.5125,170.7170,140.3945$. 
Nanoparticle based photoacoustic and photothermal contrast agents have received widespread interest for both diagnostic and therapeutic applications [1-10]. Light absorbing nanoparticles are attractive for such applications because they can exhibit large optical absorption cross-sections when excited with optical radiation in the surface plasmon absorption band, resulting in efficient and localized heating [2]. In addition, nanoparticles can be conjugated with antibodies or proteins to target specific biomarkers, opening up the possibility of molecular photoacoustic imaging or selective thermal therapy [11]. Nanoparticles used as contrast agents include gold nanospheres [1-5], nanocages [6], nanoshells [7] and nanorods [8], carbon nanotubes [9], and gold coated carbon nanotubes [10].

Transient illumination of light absorbing nanoparticles using pulsed laser sources can produce rapid and highly localized heating. If a particle absorbs a sufficient amount of laser energy, then vaporization of a layer of liquid blanketing the particle ensues and the bubble goes through an expansion phase followed by cooling and collapse [12], a process otherwise known as inertial cavitation [13]. Laser induced cavitation is accompanied by an intense acoustic emission potentially enabling, for example, improvements in the sensitivity of photoacoustic imaging of nanoparticles or the depth within scattering media at which they can be detected. In addition, the mechanical shock and high pressures associated with bubble collapse have been shown to be a primary source of cell damage in pulsed laser based photothermal therapy or nanothermolysis $[5,12]$. The threshold fluence for micro-bubble formation depends on nanoparticle type and dimensions, as well as the laser wavelength. Typically values for nanoparticles used for biological 
applications range from $\sim 300 \mathrm{~mJ} / \mathrm{cm}^{2}$ (40 $\mathrm{nm}$ diameter gold nanoparticle excited at 532 $\mathrm{nm})$ to $\sim 3.8 \mathrm{~mJ} / \mathrm{cm}^{2}(11 \mathrm{~nm} \times 99 \mathrm{~nm}$ gold coated nanotubes excited at $850 \mathrm{~nm})$ [10].

The threshold laser fluence for nanoparticle targeted bubble formation can be reduced if the nanoparticles are simultaneously subjected to an ultrasound field, and the laser pulse is timed to illuminate the nanoparticles during the rarefaction phase of the ultrasound [14, 15]. Moreover, the resulting cavity expansion is dramatically increased, resulting in greater collapse energy and more intense acoustic emissions. In this Letter, we demonstrate ultrasound assisted gold nanosphere seeded cavitation at laser fluencies well below those previously reported for similar structures. The ultrasound signal associated with bubble collapse exceeds the direct photoacoustic response, and is used to image a nanoparticle-doped region in a tissue phantom.

A schematic of the experimental set-up is shown in Figure 1. The nanoparticle doped polyacrylamide gel samples were placed in a test tank filled with degassed water. A pulsed laser source (Continuum, Minilite-10) with a pulse width of $10 \mathrm{~ns}$ and operating at a wavelength of $532 \mathrm{~nm}$ was used to illuminate the target. The beam size (full width at half maximum) was $2.38 \mathrm{~mm}$. The laser energy was controlled using a variable attenuator, which was calibrated to provide the pulse energy at the target. The target was insonified using a $1.1 \mathrm{MHz}$ single- element, high intensity focused ultrasound (HIFU) transducer (Sonic Concepts, H101) with a focal length of $63 \mathrm{~mm}$ and an ellipsoidal focal region of diameter $2 \mathrm{~mm}$ and length $19 \mathrm{~mm}(6 \mathrm{~dB})$. The pressure output of the HIFU transducer was calibrated in water using a membrane hydrophone (Precision Acoustics). 
The transducer was driven by a 3-cycle sinusoidal pulse generated by a function generator and power amplifier, combined with an impedance matching circuit. The photoacoustic signals generated at the target were detected using a $15 \mathrm{MHz}$ focused broadband transducer (Olympus NDT, V313) with a focal length of $19 \mathrm{~mm}$. The detected signals were amplified by $40 \mathrm{~dB}$ and recorded on an oscilloscope. A digital high pass filter at $5.0 \mathrm{MHz}$ was then applied to the signals to isolate the photoacoustic response from the scattered HIFU drive signal. The laser, HIFU transducer, and detection transducer were co-aligned using a $2 \mathrm{~mm}$ diameter ball bearing as an alignment target. The target was positioned in the central region of the laser beam and the transducers were positioned by optimizing the pulse-echo response.

Polyacrylamide gel spheres doped with $80 \mathrm{~nm}$ diameter gold nanospheres (British Biocell International) were fabricated with diameters ranging from 530-670 $\mu \mathrm{m}$. The spheres were fabricated using a syringe to drop beads of gel into light oil, where they were allowed to cure. After removal from the oil, the spheres were inspected and sized using an optical microscope. The nanoparticle concentration within the spheres was approximately $10^{9}$ particles per ml. An image of a typical sphere is included in Fig.1. The spheres were embedded in a clear polyacrylamide gel matrix, which was fabricated on a small rod allowing for positioning of the phantoms within the test tank using computer controlled micrometer stages. Prior to each experiment, the doped sphere was positioned within the focal region of the transducers by monitoring the photoacoustic response while irradiating the spheres with a fluence of $25.3 \mathrm{~mJ} / \mathrm{cm}^{2}$. A delay circuit was used to fire the laser pulse $42.50 \pm 0.02 \mu \mathrm{s}$ after the HIFU transducer, corresponding to the time when the 
last rarefaction cycle arrived at the target at the time of illumination. The target size was on the order of half of the HIFU wavelength such that the majority of the target was under rarefaction at the time when the laser pulse was fired. The last refraction cycle was selected in order to subject the bubbles, once formed, to a single rarefaction cycle and prevent bubble rebound and multiple growth-collapse events.

The photoacoustic signal detected from a $550 \mu \mathrm{m}$ doped sphere at a fluence of 11.4 $\mathrm{mJ} / \mathrm{cm}^{2}$ and in the absence of ultrasound is shown in Fig. 2(a). The top signal, which is offset by $15 \mathrm{mV}$, shows a single shot of raw data. The photoacoustic signal is obscured by the noise in the system. The bottom signal shows the response after 500 time-domain signal averages. The peak amplitude of the signal is $6.8 \mathrm{mV}$. Figure 2(b) shows raw single-shot signals received from the same sample under identical illumination conditions, but also subjected to ultrasound pulses with peak negative pressures of 1.5 (top signal offset by $225 \mathrm{mV}$ ) and 1.0 MPa. A strong acoustic response is observed in both traces at $13.1 \mu \mathrm{s}$, approximately $0.55 \mu$ s after the photoacoustic response seen in Fig. 2(a). The signal is due to the expansion and subsequent driven inertial collapse of micro-bubbles formed in the sphere, the latter of which occurs during the compression phase of the ultrasound field. The inertial collapse emissions have peak amplitudes of 190 $\mathrm{mV}$ and $140 \mathrm{mV}$ for driving pressures of 1.5 and $1.0 \mathrm{MPa}$, respectively. The bubble collapse signals have substantially higher amplitudes than the corresponding photoacoustic signals, and enable single-shot detection of the nanoparticle-doped region.

The peak amplitudes of the inertial collapse signals and photoacoustic signal observed as 
a function of laser fluence are shown in Fig. 2(c). The plot consists of pooled data obtained from three different nanoparticle doped spheres of diameter 530, 550 and 670 $\mu \mathrm{m}$. The inertial collapse signal was not observed on every laser shot in the low fluence regime. The inset in Fig. 2(c) gives the probability of inertial collapse event as a function of laser fluence. The threshold peak signal amplitude for definition of an inertial collapse event was taken to be $50 \mathrm{mV}$. The amplitude data shown in Fig. 2(c) for ultrasound pressures of 1.0 and 1.5 MPa include only measurements where such a collapse event was detected. The error bars indicate the standard deviation of the measurement. The data indicates that the inertial collapse amplitude is primarily dictated by the applied ultrasound field rather than the laser fluence, and that the amplitude shows relatively large fluctuations about the mean, particularly at the larger peak negative pressure amplitude. No inertial collapse signal was observed below a fluence of $4.2 \mathrm{~mJ} / \mathrm{cm}^{2}$ for either sound pressure, while the all laser pulses produced inertial collapse signals above $13.1 \mathrm{~mJ} / \mathrm{cm}^{2}$. The stochastic behaviour observed between these two values is thought to be due to spatial or temporal fluctuations in the laser pulse and jitter in the timing of the pulse with respect to the ultrasound field, either of which could potentially lead to random transitions across the cavitation threshold.

The ability to use the inertial collapse signal for imaging was also demonstrated. The nanoparticle doped sphere phantom was scanned across the zone where the laser and transducer foci overlapped in steps of $50 \mu \mathrm{m}$. At each position, ten A-scans were taken, and the peak value of the photoacoustic or inertial collapse signal from each waveform was selected from a $\pm 0.6 \mu$ s time window of where the respective arrival was expected to 
occur. The overall maximum amplitude signal was then used for imaging. This signal processing approach was used to reduce the amplitude scatter of the inertial collapse signals. Such maximum amplitude projection images of nanoparticle doped regions are shown in Fig.3, in which the peak signal observed from each set of A-scans is plotted as a function of position. Figure 3(a-b) show photoacoustic images of nanoparticle doped spheres at fluencies of $22.6 \mathrm{~mJ} / \mathrm{cm}^{2}$ (Fig. 3(a)) and $11.4 \mathrm{~mJ} / \mathrm{cm}^{2}$ (Fig. 3(b)). Note that for the smaller laser fluence the signal is below the noise level for the system. Figure 3(c-d) show images generated from the inertial collapse emissions generated in the same samples under the same laser fluences with the application of ultrasound with a peak negative pressure of $1.5 \mathrm{MPa}$. The application of the applied field results in dramatic improvement in our ability to detect the presence of nanoparticles in the phantom. It is noted that inertial collapse emissions were only observed in the presence of both ultrasound and laser fields under these experimental conditions.

In summary, this letter describes a technique for producing targeted cavitation at light absorbing nanoparticles through simultaneous excitation by pulsed optical and ultrasound fields. When combined with more efficient photothermal contrast agents, such as gold nanorods, ultrasound assisted cavitation may be suitable for therapeutic and imaging applications at depth in biological tissue.

This research was supported by the Bernard M. Gordon Center for Subsurface Sensing and Imaging Systems under EEC-9986821 and a B.U. Dean's Catalyst Award. T.W. Murray also acknowledges the support of the NSF through grant CMS-0448796. 


\section{REFERENCES}

1. A.A. Oraevsky, A.A. Karabutov, and E.V. Savateena, "Enhancement of optoacoustic tissue contrast with absorbing nanoparticles," Proc. SPIE 4434, 60-69 (2001).

2. X. Huang, P.K. Jain, I.H. El-Sayed, and M.A. El-Sayed, "Plasmonic photothermal therapy (PPTT) using gold nanoparticles," Lasers Med. Sci. 23, 217-228 (2008).

3. C.M. Pitsillides, E.K. Joe, X. Wei, R.R. Anderson, and C.P. Lin, "Selective cell targeting with light-absorbing microparticles and nanoparticles," Biophys. J. 84, 4023$4032(2003)$.

4. V.P. Zharov, V. Galitovsky, M. Viegas, "Photothermal detection of local thermal effects during selective nanothermolysis, Appl. Phys. Lett. 83, 4897-4899 (2003).

5. V.P. Zharov, K.E. Mercer, E.N. Galitovskaya, and M.S. Smeltzer, "Photothermal nanotherapeutics and nanodiagnostics for selective killing of bacteria targeted with gold nanoparticles,” Biophys. J. 90(2) 619-627 (2006).

6. X.M. Yang, S.E. Skrabalak, Z. Li, Y. Xia, and L.V. Wang, "Photoacoustic tomography of a rat cerebral cortex in vivo with Au nanocages as an optical contrast agent," Nano Letters 7(12), 3798-3802 (2007).

7. Y. Wang, X. Xie, X. Wang, G. Ku, K. L. Gill, D. P. O’Neal, G. Stoica, and L. V. Wang, "Photoacoustic tomography of a nanoshell contrast agent in the in vivo rat brain," Nano Letters 4(9), 1689-1692 (2004).

8. M. Eghtadri, A. Oraevsky, J.A. Copland, N.A. Kotov, A. Conjusteau, and M. Motamedi, "High sensitivity in vivo detection of gold nanorods using a laser optoacoustic imaging system," Nano Lett. 7(7), 1914-1918 (2007). 
9. J-W. Kim, E.V. Shashkov, E.I. Galanzha, N. Kotagiri, and V.P. Zharov, "Photothermal antimicrobial nanotherapy and nanodiagnostics with self assembling carbon nanotube clusters", Lasers Surg. Med. 39, 622-634 (2007).

10. J-W. Kim, E.I. Galanzha, E.V. Shashkov, H-M. Moon, and V.P. Zharov, "Golden carbon nanotubes as multimodal photoacoustic and photothermal high contrast molecular agents," Nature Nanotech. 4, 688-694 (2009).

11. M. Ferrari, "Cancer nanotechnology: Opportunities and challenges," Nat. Rev. Cancer 5, 161-171 (2005).

12. D. Lapotko, "Optical excitation and detection of vapor bubbles around plasmonic nanoparticles", Opt. Express, 17(4), 2538-2556 (2009).

13. T.G. Leighton, The Acoustic Bubble, (Academic Press, 1994).

14. C.H. Farny, T. Wu, R.G. Holt, T.W. Murray and R.A. Roy, "Nucleating cavitation from laser-illuminated nano-particles," Acoust. Res. Lett. Online 6(3), 138-143 (2005).

15. B. Krasovitski, H. Kislev, and E. Kimmel, "Modeling photothermal and acoustical induced bubble generation and growth," Ultrasonics 47, 90-101 (2007). 


\section{FIGURE CAPTIONS}

Fig. 1. Experimental setup. The following abbreviations are used: IMB- impedance matching box; FLS- flashlamp sync; PBS- polarizing beamsplitter; and PDphotodetector.

Fig. 2. (a) Single shot (top) and averaged (500x) photoacoustic signals generated at a fluence of $11.4 \mathrm{~mJ} / \mathrm{cm}^{2}$. (b) Single shot inertial collapse signals generated at the same fluence as (a) but with 1.0 and 1.5 MPa ultrasound fields. (c) Peak amplitudes of inertial collapse signals and photoacoustic (PA) signals generated as a function of laser fluence. The inset shows the probability of detecting an inertial collapse event.

Fig. 3. Images of nanoparticle doped spheres generated from the photoacoustic response to a laser fluence of (a) $22.6 \mathrm{~mJ} / \mathrm{cm}^{2}$ and (b) $11.4 \mathrm{~mJ} / \mathrm{cm}^{2}$. Images created using the inertial collapse signals with an applied 1.5 MPa ultrasound field are shown in (c) for a laser fluence of $22.6 \mathrm{~mJ} / \mathrm{cm}^{2}$ and (d) for $11.4 \mathrm{~mJ} / \mathrm{cm}^{2}$. The sphere diameters were $530 \mu \mathrm{m}$ $(\mathrm{a}, \mathrm{c})$ and $550 \mu \mathrm{m}(\mathrm{b}, \mathrm{d})$. 
Figure 1

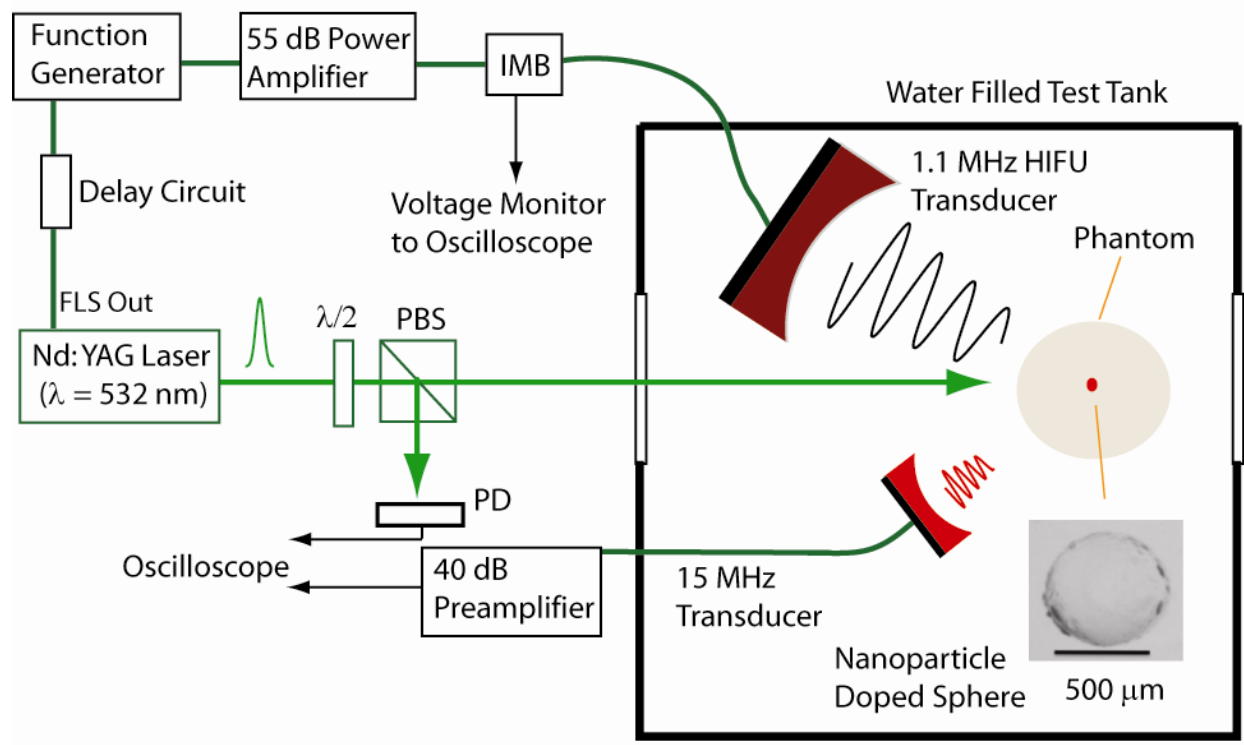


Figure 2
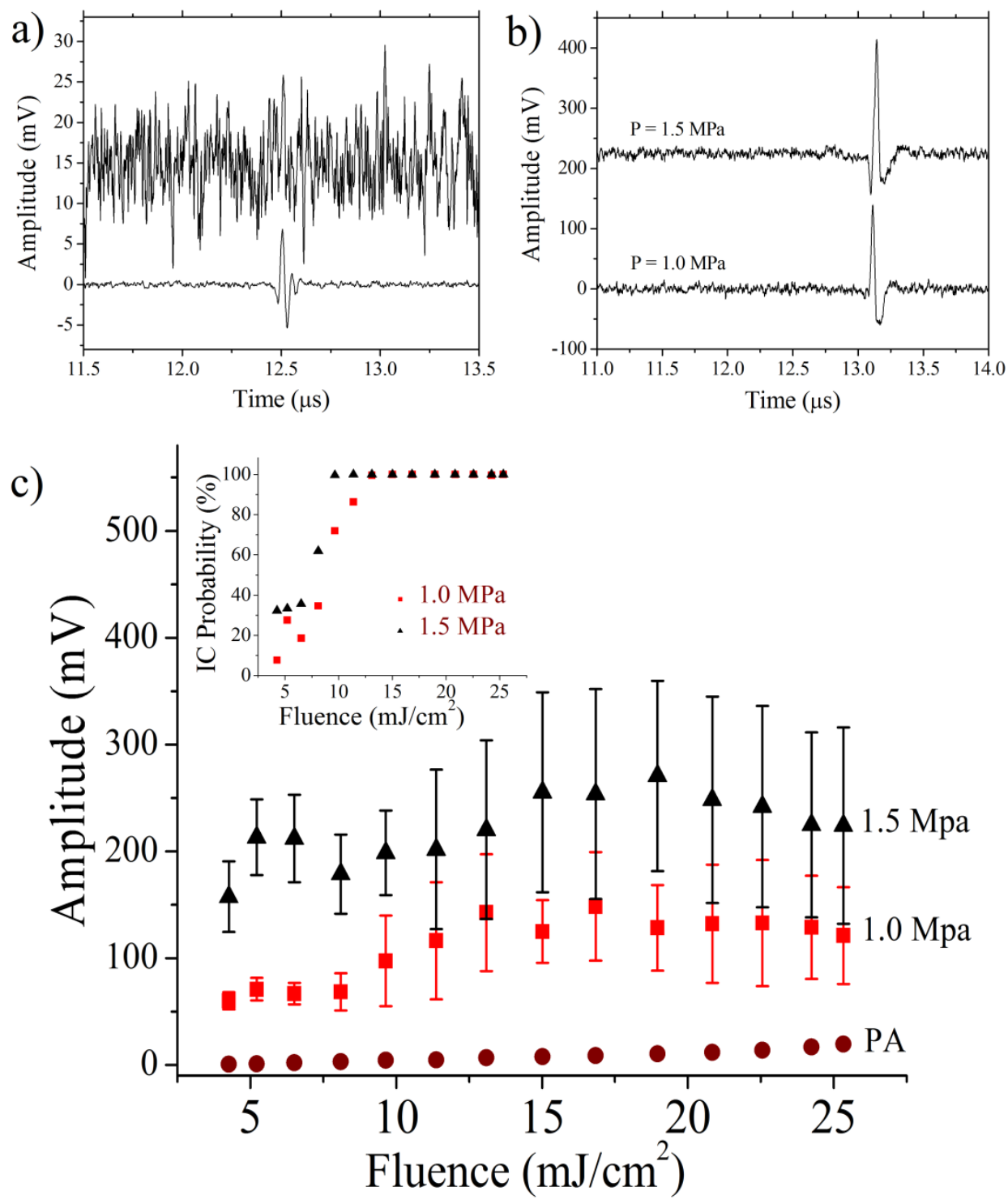
Figure 3.
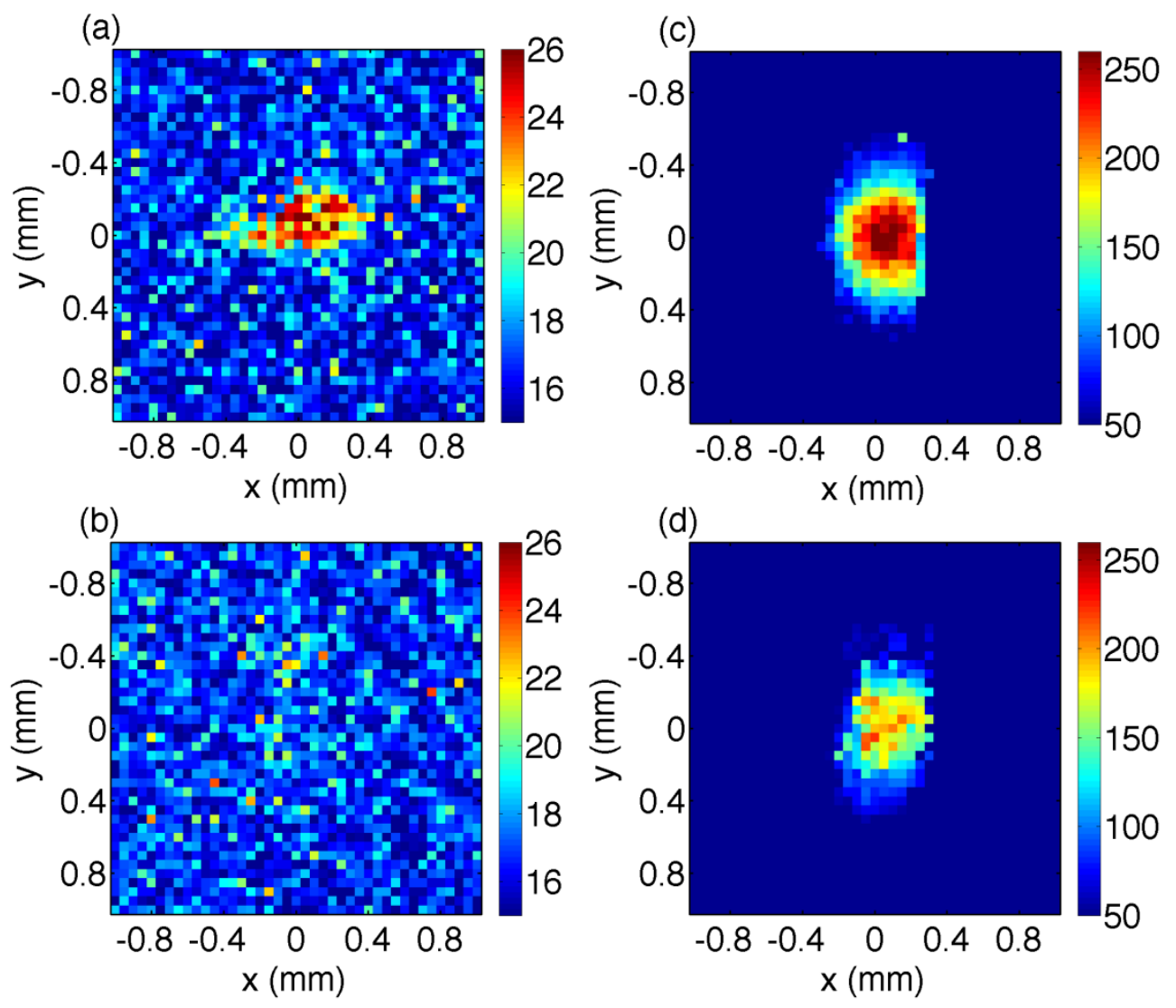